\title{
小麦中一个PDR型ABC转运蛋白基因的克隆和 特征分析
}

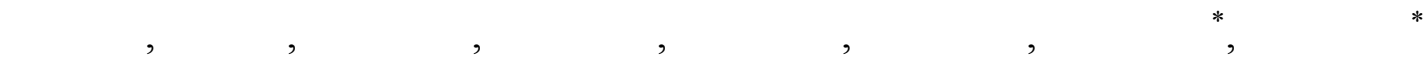

(1) 南京农业大学作物遗传与种质创新国家重点实验室, 细胞遗传研究所, 南京 210095

(2) 浙江省农业科学院作物与核技术利用研究所, 杭州 310021

*联系人, E-mail: xiuew@njau.edu.cn; djliu@njau.edu.cn

2009-03-21 收稿, 2009-06-06 接受

国家自然科学基金(批准号: 30330380)、高等学校创新引智计划(批准号: B08025)、国家高技术研究发展计划 (编号: 2006AA10Z1F6)、江苏省 自然科学基金重点项目(批准号: BK2006720)、国家转基因生物新品种培育科技重大专项(编号: 2009ZX08002-001B)及美国 McKnight 基金 CCRP 资助项目

摘要 脱氧血腐镰刀菌烯醇(deoxynivalenol, DON)在小麦赤霉病发病过程中起重要 作用, 是一种毒性因子. 禾谷镰刀菌的侵染能力依赖于其产生 DON 的能力, 抗病品 种能显著降低病穗组织中 DON 的含量. 本研究利用 Affymetrix 小麦基因组芯片, 对 抗赤霉病小麦品种望水白经 DON 诱导后的穗组织基因表达特点进行了分析, 结果 发现, 一个编码 PDR 型转运蛋白的 EST 受 DON 诱导后上调表达 45 倍. 根据该 EST 设计引物篮选小麦基因组 TAC 文库, 得到一个包含该基因的 TAC 单克隆. 利用染 色体 walking 对该单克隆测序，用 Softberry 软件进行基因预测，根据预测基因的 $5^{\prime}$ 和 $3^{\prime}$ 非翻译区设计引物, 从 DON 诱导的小麦望水白穗组织 cDNA 中克隆出该转运 蛋白基因. 该基因组全长 $7377 \mathrm{bp}$, 包含 19 个外显子, CDS 长度为 $4308 \mathrm{bp}$, 编码长 $1435 \mathrm{aa}$ 且分子量 $161 \mathrm{kD}$ 的蛋白. 蛋白序列比对表明, 该基因属于PDR 蛋白家族, 命 名为 TaPDR1 (Triticum asetivum Pleiotropic Drug Resistance). 利用一套中国春缺体四体系将 TaPDR1 基因定位在小麦 5A 染色体上. 半定量 RT-PCR 表明, TaPDR1 在 望水白穗中受 $\mathrm{DON}$ 和禾谷镰刀菌诱导表达, 表明其参与了植物抗病防御反应. 该基 因在望水白感病突变体中低水平表达, 进一步证明 TaPDR1 与赤霉病抗性有关. TaPDR1 的表达不受与生物胁迫相关的激素(JA 和 SA)和非生物胁迫因子(热、冷、 伤害和 $\mathrm{NaCl}$ )的诱导, 但受到 $\mathrm{Al}^{3+}$ 和游离 $\mathrm{Ca}^{2+}$ 的诱导表达, 推测 $\left[\mathrm{Ca}^{2+}\right]_{\mathrm{i}}$ 介导了 TaPDR1 的表达信号.

小麦赤霉病(FHB)是一种由禾谷镰刀菌(Fusarium graminearum)引起的世界性病害, 该病发生后不仅降 低作物产量，而且病麦粒中含有多种危害人、畜健康 的单端孢霉烯族毒素(trichothecene toxins), 影响粮食 品质 ${ }^{[1]}$. 其中脱氧血腐镰刀菌烯醇(DON)是粮食中最
关键词

普通小麦

脱氧血腐镰刀菌烯醇(DON)

$\mathrm{ABC}$ 转运蛋白

PDR 
养型, 然后转向坏死营养阶段, 其产生和积累的 DON等单族毒素在转变过程中起重要作用. Mesterhazy等人 ${ }^{[6]}$ 研究表明, 禾谷镰刀菌的侵染能力依赖于 其产生DON的能力, FHB的田间发病程度与麦粒或病 穗组织中DON含量之间有极显著的相关性, DON单独 处理即可引起较典型的赤霉病症状. 抗病品种能显 著地降低病穗中DON的含量, 因而具有不同FHB抗 性的品种其籽粒中DON含量也有很大的差别 ${ }^{[7.87}$. 自 发现抗病品种能够阻止DON毒素合成或促使其降解 之后, 在这方面做了很多研究工作, 目前已经发现了 一些和抗毒素或降解毒素积累相关的基因, 如具有降 解DON功能的小麦乙酰基转移酶基因 ${ }^{[9]}$ 、具有耐DON 功能的水稻核糖体蛋白基因 $\mathrm{L} 3(R P L 3)^{[10]}$ 和小麦中核 糖体蛋白基因 $\mathrm{L} 3(R P L 3)^{[111}$ 、具有降解DON功能的拟南 芥UDP-葡萄糖基转移酶基因DOGT1 $1^{[12]}$ 和具有转运 DON功能的酵母(Saccharomyces cerevisiae) $\mathrm{ABC}$ 转运 蛋白基因 $S C P D R 5^{[12,13]}$. 研究表明, 位于小麦 $3 \mathrm{BS}$ 上的 抗赤霉病和抗DON的主效QTL (FHB1)对DON的抗性 有贡献 ${ }^{[14 ~ 17]}$, 它可能是通过编码或调控DON脱毒基因 实现对DON的抗性 ${ }^{[18]}$. 由于小麦抗 DON 是个复杂的 数量性状, 而且小麦抗赤霉病和抗DON的机理还不清 楚, 所以鉴定一些新的与小麦抗DON反应密切相关的 基因显得尤为重要.

小麦地方品种望水白是到目前公认的抗赤霉病 性最好、抗性最稳定、DON含量也很低的一个品种 [19-21]. 在望水白中已经鉴定并定位了一些与赤霉病 抗性和DON抗性相关的主效QTL，如FHB1 ${ }^{[16,22,23]}$. 本 研究利用Affymetrix 小麦基因芯片对望水白经DON 诱导后穗组织基因表达特点进行了分析, 结果发现, 小麦中的一个PDR型 $\mathrm{ABC}$ 转运蛋白基因受到DON的 诱导上调表达, 我们从望水白中克隆出这个转运蛋 白基因, 该基因不但受到DON和禾谷镰刀菌的诱导 表达, 还受到FHB1 的调控, 显示该基因在抗赤霉病 中发挥作用.

\section{1 材料与方法}

（i ）材料. 抗赤霉病小麦品种望水白、望水白 感赤霉病突变体(该突变体是从快中子处理的望水白 后代群体中获得的, 表现稳定的感赤霉病特性, 突变 体的获得和鉴定结果将另文发表)、感赤霉病小麦品 种 Alondra's 和小麦品种中国春的缺体-四体系列、小
麦基因组 TAC 文库等均由本实验室提供.

(ii ) TaPDR1 基因的克隆. 根据小麦芯片杂交 结果信息上调表达的探针所对应的 EST (BQ161629) 序列设计特异引物 SH49S (5'-ACTTTGACAACAACCGACAG-3') 和 SH49A (5'-GCATTAAGCACGATTTCC$\left.3^{\prime}\right)$, 筛选小麦基因组 TAC 文库. 以该 TAC 文库为模 板进行 PCR 反应. 反应体系为: TAC 文库菌液 $2 \mu \mathrm{L}$, $10 \mu \mathrm{mol} / \mathrm{L} \mathrm{SH} 49 \mathrm{~S}$ 引物和 $\mathrm{SH} 49 \mathrm{~A}$ 引物各 $0.5 \mu \mathrm{L}$; $10 \times \mathrm{PCR}$ 缓冲液 $2.5 \mu \mathrm{L} ; 2.5 \mathrm{mmol} / \mathrm{L}$ dNTP $2.0 \mu \mathrm{L} ; 25$ $\mathrm{mmol} / \mathrm{L} \mathrm{Mg}^{2+} 1.5 \mu \mathrm{L} ; 5 \mathrm{U} / \mu \mathrm{L}$ Taq 聚合酶 $0.2 \mu \mathrm{L}$, 加水 至总体积 $25 \mu \mathrm{L}$. 反应条件为: $94^{\circ} \mathrm{C}, 3 \mathrm{~min} ; 94^{\circ} \mathrm{C}, 30 \mathrm{~s}$, $55^{\circ} \mathrm{C}, 45 \mathrm{~s}, 72^{\circ} \mathrm{C}, 50 \mathrm{~s}, 33$ 个循环; $72^{\circ} \mathrm{C}$ 延伸 $10 \mathrm{~min}$. PCR 产物用 $8 \%$ PAGE 胶电泳, 银染检测, 对篮选到 的阳性单克隆提取质粒并用染色体步行法对单克隆 测序. 用 Softberry 软件(http://www.softberry.com)对 序列进行结构分析. 根据序列预测结果, 在目标基因 的 $5^{\prime}$ 和 $3^{\prime}$ 非翻译区各设计一对引物 CPDR1S (5'-CACGGATCCACAGACAGGCACAGAAAGA-3') 和 CPDR1A (5'-AAACCCGGGTTGACAACAACCGACAGA-3'), 从 DON 诱导的望水白穗组织 CDNA 中扩增. 反应体系: 5 $\mu \mathrm{L}$ cDNA 模板; $10 \mu \mathrm{mol} / \mathrm{L}$ CPDR $1 \mathrm{~S}$ 引物和 CPDR $1 \mathrm{~A}$ 引物各 $2 \mu \mathrm{L}, 2 \times \mathrm{GC}$ 缓冲液 $॥ 25 \mu \mathrm{L}, 2.5 \mathrm{mmol} / \mathrm{L}$ $\mathrm{dNTP} 8 \mu \mathrm{L}, 5 \mathrm{U} / \mu \mathrm{L}$ LA Taq 聚合酶 $0.5 \mu \mathrm{L}$, 加水至总体 积 $50 \mu \mathrm{L}$. 反应条件为: $94^{\circ} \mathrm{C}, 3 \mathrm{~min} ; 94^{\circ} \mathrm{C}, 30 \mathrm{~s}, 58^{\circ} \mathrm{C}$, $45 \mathrm{~s}, 72^{\circ} \mathrm{C}, 4 \mathrm{~min}, 33$ 个循环; $72^{\circ} \mathrm{C}$ 延伸 $10 \mathrm{~min}$. $\mathrm{PCR}$ 产物构建到 pGEM-T 载体(Promega 公司), 篮选到的 阳性单克隆菌斑进行扩大培养后提取质粒 DNA 并测 序.

(iii) 半定量 RT-PCR 分析. (1) DON 和禾谷镰 刀菌处理幼穗. 小麦望水白、望水白感病突变体和 Alondra's 幼穗期(正常条件大田中生长)用 DON 水溶 液 $(100 \mathrm{mg} / \mathrm{L})$ 和禾谷镰刀菌狍子 $(50 \mu \mathrm{L})$ 对幼穗进行单 花滴注法接种，于接种后不同时间段分别取幼穗，液 氮中保存待用. (2) SA, JA, 热, 冷和伤害处理叶片. 小麦望水白种子经 $75 \%$ 乙醇表面消毒 $30 \mathrm{~s}$ 后, 以 $10 \%$ 次氯酸钠消毒 $10 \mathrm{~min}$, 无菌水洗 3 次, 黑暗条件下发 芽 $2 \mathrm{~d}$, 转入 $16 \mathrm{~h}$ 光照 $8 \mathrm{~h}$ 黑暗光周期下生长, 每天换新 鲜的无菌水, 生长 2 周后对叶片分别进行以下处理: 叶 面喷施 $1 \mathrm{mmol} / \mathrm{L} \mathrm{SA}$ 或 $0.1 \mathrm{mmol} / \mathrm{L} \mathrm{JA}$, 取处理后不同时 间段的叶片, 液氮中保存待用; 取生长 2 周的幼苗, 分别 转移到 $40^{\circ} \mathrm{C}$ 的人工气候箱中处理 $1 \mathrm{~h}$ 或 $4^{\circ} \mathrm{C}$ 的人工气候 
箱中处理, 取处理后不同时间段的叶片, 液氮中保存 待用; 取生长 2 周的幼苗, 对叶片进行机械伤害, 取 处理后不同时间段的叶片, 液氮中保存待用. (3) $\mathrm{NaCl}$ 处 理根. 将正常生长 $3 \mathrm{~d}$ 的望水白幼苗, 转入 $300 \mathrm{ng} / \mathrm{mL}$ 的 DON 溶液中处理不同时间段后取根, 液氮中保存待 用. (4) $\mathrm{Al}^{3+}$ 和 $\mathrm{Ca}^{2+}$ 处理根. 望水白种子经 $75 \%$ 乙醇 表面消毒 $30 \mathrm{~s}$ 后, 以 $10 \%$ 次氯酸钠消毒 $10 \mathrm{~min}$, 无菌 水洗 3 次, 于 $0.5 \mathrm{mmol} / \mathrm{L} \mathrm{CaCl}_{2}$ 溶液中 $(\mathrm{pH} \mathrm{4.5)}$ 、黑暗 条件下发芽 $2 \mathrm{~d}$, 转入 $16 \mathrm{~h}$ 光照 $/ 8 \mathrm{~h}$ 黑暗光周期下生 长, 每天换新鲜的 $0.5 \mathrm{mmol} / \mathrm{L} \mathrm{CaCl}_{2}$ 溶液 ( $\left.\mathrm{pH} 4.5\right)$, 生 长 $3 \mathrm{~d}$ 后, 分别换成包含 $0,10 ， 20 ， 50 ， 100$ 和 200 $\mu \mathrm{mol} / \mathrm{L} \mathrm{AlCl}_{3}$ 的 $0.5 \mathrm{mmol} / \mathrm{L} \mathrm{CaCl}_{2}$ 溶液 $(\mathrm{pH} 4.5$ ) 和 0.05 $\mathrm{mmol} / \mathrm{L} \mathrm{CaCl}_{2}$ 溶液中 $(\mathrm{pH} 4.5)$, 取处理 $6 \mathrm{~h}$ 的根, 液氮 中保存待用. (5) RT-PCR. 提取备用材料的 RNA, 用 AMV Reverse Transcriptase XL (TaKaRa 公司)反转录 成 cDNA. 以 cDNA 为模板、SH49S 和 SH49A 为引 物、管家基因 Tubulin 为内参(扩增 Tubulin 基因的引 物为 tubulin F: 5'-CTCATCACAGGCAAGG- AAGAT-3' 和 tubulin R: 5'-TTAAGGTAAGTGTAGGTTGGG-3')进行 RT-PCR, 分析 TaPDR1 的表达情况. 反应体系: $2 \mu \mathrm{L} \mathrm{cDNA}$ 为模板, $10 \mu \mathrm{mol} / \mathrm{L} \mathrm{SH} 49 \mathrm{~S}$ 引物 和 $\mathrm{SH} 49 \mathrm{~A}$ 引物各 $0.5 \mu \mathrm{L}, 10 \times \mathrm{PCR}$ 缓冲液 $2.5 \mu \mathrm{L} ; 2.5$ $\mathrm{mmol} / \mathrm{L}$ dNTP $2 \mu \mathrm{L} ; 25 \mathrm{mmol} / \mathrm{L} \mathrm{Mg}^{2+} 1.5 \mu \mathrm{L} ; 5 \mathrm{U} / \mu \mathrm{L}$ $T a q$ 聚合酶 $0.2 \mu \mathrm{L}$, 加水至总体积 $25 \mu \mathrm{L}$. 反应条件为: $94^{\circ} \mathrm{C}, 3 \mathrm{~min} ; 94^{\circ} \mathrm{C}, 30 \mathrm{~s}, 55^{\circ} \mathrm{C}, 45 \mathrm{~s}, 72^{\circ} \mathrm{C}, 50 \mathrm{~s}, 28$ 个循 环; $72^{\circ} \mathrm{C}$ 延伸 $10 \mathrm{~min}$. 扩增 Tubulin 基因的引物退火温 度为 $53^{\circ} \mathrm{C}$, 其他条件相同.

(iv) 系统进化树构建. 使用MEGA4 ${ }^{[24]}$ 软件以 邻接法(Neighbor-Joining, NJ)构建系统发生树. 系统 树中分支的置信水平用自引导值(Bootstrap test)估计,
重复 1000 次.

（v）染色体定位. 用一套普通小麦品种中国春 的缺体-四体材料基因组 DNA 为模板进行 PCR 扩增, 对 TaPDR 在小麦中进行染色体定位, 染色体定位使 用的引物为 SH49S 和 SH49A.

\section{2 结果和分析}

\subsection{TaPDR1 基因的克隆}

根据小麦芯片杂交中上调 45 倍的探针所对应的 EST(BQ161629: 一个 ABC 转运蛋白同源基因)序列 设计特异引物 SH49S 和 SH49A, 篮选小麦基因组 $\mathrm{TAC}$ 文库, 对篮选到的阳性单克隆菌斑进行扩大培 养后提取质粒 DNA 并测序, 用 Softberry 软件进行预 测, 根据预测基因的 5 '和 $3^{\prime}$ 非翻译区设计引物, 从 DON 诱导的小麦望水白穗组织 cDNA 中克隆出这个 转运蛋白基因, 其基因组全长 $7377 \mathrm{bp}$, 含 19 个外显 子, CDS 长度为 $4308 \mathrm{bp}$, 编码一个 1435 aa 且分子量 $161 \mathrm{kD}$ 的蛋白(图 1). 用该基因的蛋白序列在 NCBI 上进行结构域比对, 该基因属于 PDR 亚家族(图 2), PDR 转运蛋白的结构特征是核酸结构域(nucleotide binding domains)比跨膜结构域(transmem- brane domains)更接近序列的 $\mathrm{N}$ 端, 所以把该基因定名为 TaPDR1(Triticum asetivum Pleiotropic Drug Resistance), $T a P D R 1$ 的 cDNA 和基因组序列在 GenBank 上的登录 号分别为 FJ185035 和 FJ858380.

\section{2 同源性比较}

植物PDR 基因分为 5 个族 ${ }^{[25]}, T a P D R 1$ 属于第 4 族(图 3). 与 TaPDR1 同源性最近的是水稻的OsPDR3, OsPDR4 和OsPDR5, 氨基酸一致性分别为 79\%, 71\% 和 $68 \%$, 该基因与拟南芥的AtPDR6 和AtPDR11 均有

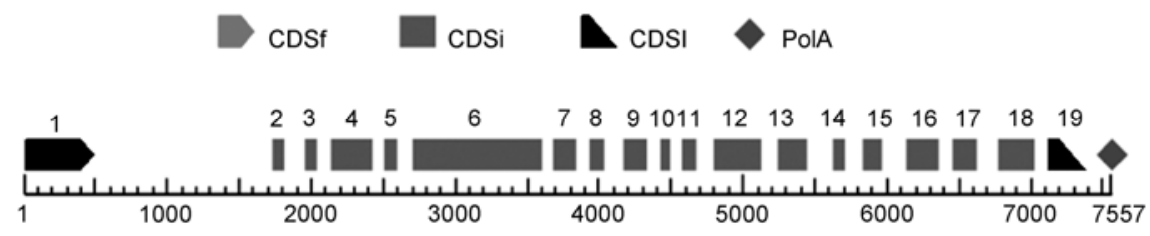

图 1 TaPDR1 的基因组组织结构

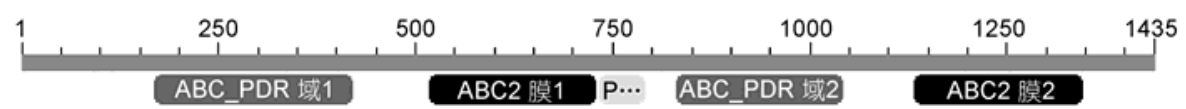

图 2 TaPDR1 蛋白的结构域 


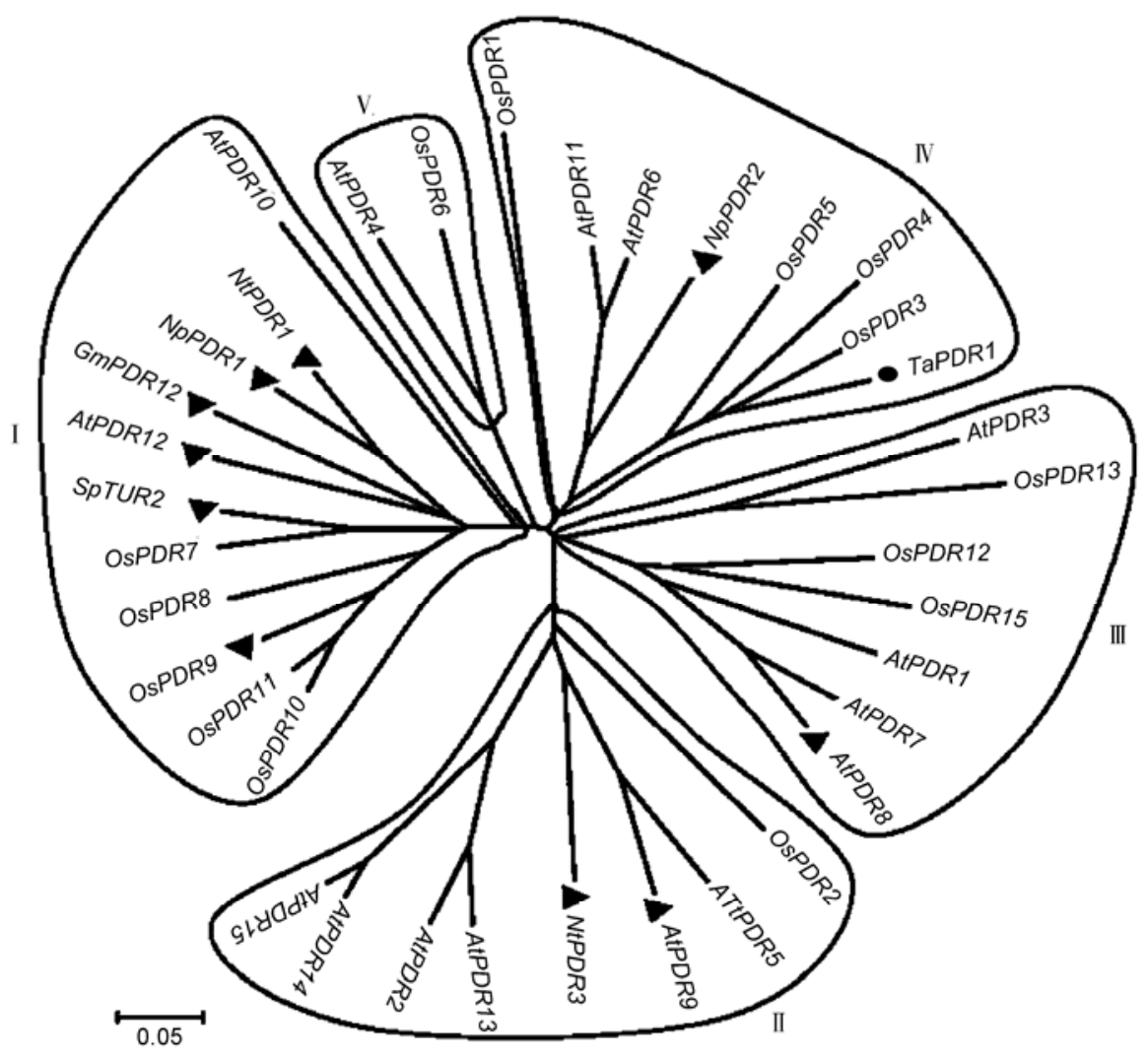

图 3 植物 PDR 基因的系统进化

三角形表示已经有功能报道的转运蛋白, 圆圈表示本实验克隆的基因

$61 \%$ 的氨基酸一致性. 到现在为止，末见上述水稻和 拟南芥的同源基因相关功能研究的报道, 烟草的 $N p P D R 2$ 是这一族中唯一有功能研究的基因 ${ }^{[26]}$. $N P P D R 2$ 和TaPDR 1 具有 $61 \%$ 的氨基酸一致性, 与其 他的PDR基因不同, NpPDR2 在叶片中不被任何一种 与生物或非生物胁迫反应相关的激素所诱导, 而且 通过RNA干扰, 阻止NpPDR2 的表达, 未观察到植物 出现任何不同的表型. 所以, 这一族PDR基因在植物 体中的功能尚不明了.

\subsection{TaPDR1 在小麦染色体上的定位}

利用引物 SH49S 和 SH49A 在一套普通小麦品种 中国春的缺体-四体材料的基因组 DNA 中扩增, 对 $T a P D R 1$ 进行染色体定位. 结果表明, 在缺失 $4 \mathrm{~A}, 5 \mathrm{~A}$ 和 $5 \mathrm{D}$ 染色体的材料中各缺失了一条扩增带(图 4). 根 据 TAC 克隆测序的结果可知, 引物 SH49S 和 SH49A 可从 TaPDR1 的基因组序列上扩增出 $269 \mathrm{bp}$ 大小的片 段, 这与 $5 \mathrm{~A}$ 上缺失的片段大小一致, 所以将 TaPDR1 定位于 $5 \mathrm{~A}$ 染色体上. 小麦是一个异源六陪体, 许多基
因在 3 个染色体组上都有相同的拷贝，如图所示， TaPDR1 基因也有 3 个拷贝, 但在 DON 诱导的小麦穗 部 cDNA 中也只扩增出一条 $269 \mathrm{bp}$ 大小的片段, 而没 有检测到另外的两个片段, 表明在小麦多倍体形成时, 发生了遗传二倍体化, 另两个拷贝不再表达或者发生 了功能趋异化, 不参与 DON 的抗性.

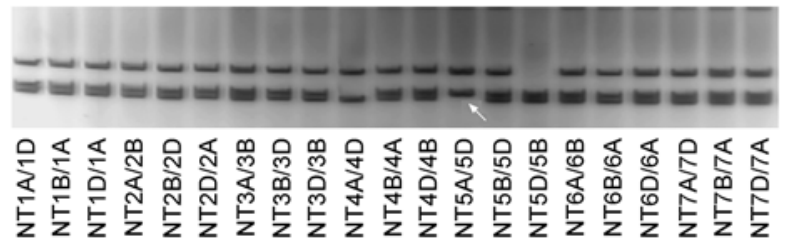

图 4 TaPDR1 的中国春缺体-四体和染色体定位 箭头所指为 $T a P D R 1$ 定位的染色体

\subsection{TaPDR1 诱导表达分析}

(i) DON 和禾谷镰刀菌对 TaPDR1 的诱导. TaPDR1 的表达受到 DON 的诱导, 在抗感品种中均 有表达, 但表达模式不同(图 5). 在抗病品种望水白 


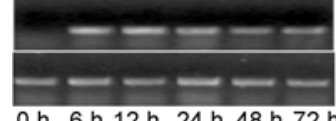

(a)

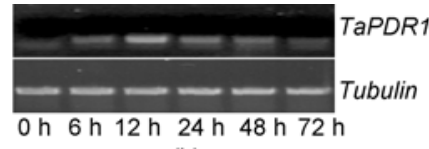

(b)
图 $5 T$ TaPDR1 的表达在抗感品种中受到 DON 的诱导 (a) 望水白; (b) Alondra's

中, 经 DON 诱导后 6 12 h TaPDR1 的表达水平达到 高峰, 然后开始缓慢下降; 而在感病品种 Alondra's 中, 直到接种后 $12 \mathrm{~h}$ 表达才达到高峰.

TaPDR1 同样可被禾谷镰刀菌所诱导表达，望水 白在接种禾谷镰刀菌 $48 \mathrm{~h}$ 可以检测到TaPDR1 的表达 (图 6). 据报道, 在接种禾谷镰刀菌 $36 \mathrm{~h}$ 后可以检测 到 $D O N^{[27]}$, 所以推测TaPDR1 在抗赤霉病中的功能更 多是与DON相关. FHB1 是定位于望水白 3BS染色体 0.78 0.87 区段一个抗赤霉病和抗DON的主效QTL, 我们利用本实验室鉴定的一个该区段缺失的感病突 变体分析 TaPDR 1 和FHB1 的关系, 结果发现, 接种禾 谷镰刀菌后感病突变体中 $T a P D R 1$ 的表达模式与在抗 病野生型中明显不同, 望水白接种禾谷镰刀菌 $48 \mathrm{~h}$ 后, $T a P D R 1$ 的表达达到最高, 而在突变体中, TaPDR 1 的 表达一直很弱, 没有检测到强烈上调(图 6), 据此推 测TaPDR1 与赤霉病或DON抗性有关. 最近报道的小 麦中一个PDR型的 $\mathrm{ABC}$ 转运蛋白基因与 $T a P D R 1$ 有 $46 \%$ 的氨基酸一致性，该基因可以持久抵抗多种小麦 真菌病害, 包括抗叶锈病的 $L r 34$, 抗条锈病的 $Y r 18$, 抗白粉病的 $P m 38$ 以及抗干叶尖的 $L t n 1$, 是一个多效 基因 ${ }^{[28]}$, 由此可见小麦 $P D R$ 基因在抗真菌性病害中 发挥重要的作用.

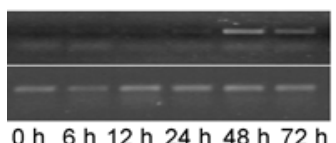

(a)

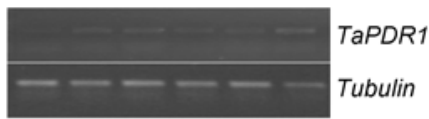

0 h 6 h 12 h 24 h 48 h 72 h

(b)
图 6 TaPDR1 的表达受到禾谷镰刀菌的诱导

(a) 望水白; (b) 感病突变体

(ii ) TaPDR1 不被SA和JA以及其他胁迫诱导. 植 物PDR基因广泛参与了植物对外界环境中生物和非生 物胁迫的应答 ${ }^{[25]}$, 但本研究表达谱显示, TaPDR1 的表 达不受到与生物胁迫相关的激素(如JA和SA)、非生物 胁迫因子(如热、冷、伤害和 $\mathrm{NaCl}$ )的诱导(结果未显示). 这一结果与 NpPDR2 的表达谱相同 ${ }^{[26]}$. 拟南芥AtPDR6
仅在根中表达，在幼苗中受到表油菜素内酯，2.4-D， $\mathrm{NaCl}, \mathrm{CdCl}, 11^{\circ} \mathrm{C}$ 低光照和 $4^{\circ} \mathrm{C}$ 黑暗的诱导, 但不受放 线酮和ABA的诱导. 但AtPDR11 不在根、茎、叶、花 和幼苗中表达 ${ }^{[29]}$. 这些结果表明第 4 族的PDR 基因具 有不同的生物学功能.

(iii) TaPDR1 受到 $\mathrm{Al}^{3+}$ 和 $\left[\mathrm{Ca}^{2+}\right]$ 的诱导. TaMDR1 是小麦根中一个受铝诱导的基因 ${ }^{[30]}$, 在本实验的芯片 结果中发现, TaMDR1 也上调表达, 表明TaMDR1 可能 与DON抗性有关. 为了研究是否TaPDR1 同样也受到 铝的诱导, 本研究利用 $\mathrm{Al}^{3+}$ 和 $\left[\mathrm{Ca}^{2+}\right]$ 对小麦根进行了 诱导, 结果表明, TaPDR1 受到 $\mathrm{Al}^{3+}$ 和 $\left[\mathrm{Ca}^{2+}\right]$ 降低的诱 导表达(图 7). 这一结果表明, TaPDR1 可能是TaMDR1 的功能同源基因, 就像酵母的PDR5 基因被认为是人 类 $M D R 1$ 基因的功能同源基因 ${ }^{[31]}$.

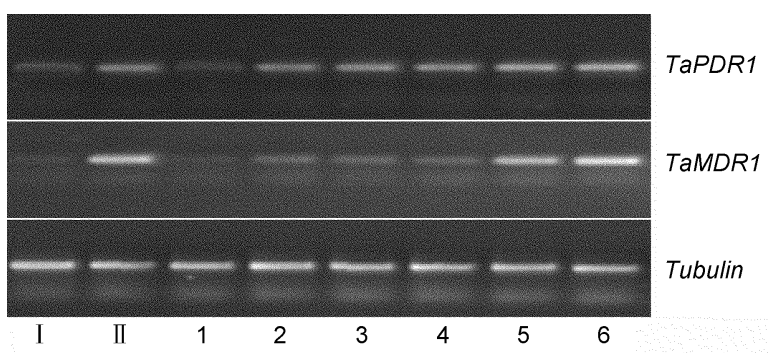

图 $7 \quad \mathrm{Al}^{3+}$ 和 $\left[\mathrm{Ca}^{2+}\right]$ 对小麦根中 TaPDR1 的诱导 在新鲜的 $0.5 \mathrm{mmol} / \mathrm{L} \mathrm{CaCl}_{2}(\mathrm{pH} 4.5)$ 溶液中生长 $3 \mathrm{~d}$ 后分别换成: I, $0.5 \mathrm{mmol} / \mathrm{L} \mathrm{CaCl}$ 溶液; $1 \mathrm{I}, 0.05 \mathrm{mmol} / \mathrm{L} \mathrm{CaCl}_{2}$ 溶液; 1 ： 含 0,10 , $20,50,100$ 和 $200 \mathrm{mmol} / \mathrm{L} \mathrm{AlCl}_{3}$ 的 $0.5 \mathrm{mmol} / \mathrm{L} \mathrm{CaCl}_{2}$ 溶液, 诱导 $6 \mathrm{~h}$

$\mathrm{Ca}^{2+}$ 是细胞内的第二信使，涉及到细胞对外界 生物和非生物胁迫反应信号的传导. 细胞受到外源 激素 $(\mathrm{JA}$ 或 $\mathrm{SA}$ )或非生物胁迫 (热、冷、伤害和 $\mathrm{NaCl}$ ) 处理时, $\left[\mathrm{Ca}^{2+}\right]_{\mathrm{i}}$ 就会增加 ${ }^{[32 \sim 37]}$. 而且这些处理均不能 诱导TaPDR1 的表达. 相反, 由于 $\mathrm{Al}$ 是钙离子通道的 抑制剂, 受到 $\mathrm{Al}$ 胁迫时使 $\left[\mathrm{Ca}^{2+}\right]_{\mathrm{i}}$ 降低; 当细胞外 的 $\left[\mathrm{Ca}^{2+}\right]$ 降低也会使 $\left[\mathrm{Ca}^{2+}\right]_{\mathrm{i}}$ 降低; 虽然在植物中没有 $\mathrm{DON}$ 和钙离子通道关系的报道，但在动物中发现 DON对心肌细胞的钙离子通道有抑制作用 ${ }^{[38,39]}$, 推 测在植物中DON处理也会使 $\left[\mathrm{Ca}^{2+}\right]_{\mathrm{i}}$ 降低. 而且这 3 种 处理均可以诱导TaPDR1 的表达. 因此推测 $\left[\mathrm{Ca}^{2+}\right]_{\mathrm{i}}$ 可 能介导了 TaPDR1 表达的信号传导.

\section{3 讨论}

ATP结合结构域(ATP-binding cassette, ABC)转运 蛋白是一类跨膜运输蛋白, 是目前已知最大、功能最广 
泛的蛋白家族, 此类蛋白的共同点是序列上含有 $\mathrm{ABC}$. 大多数 $\mathrm{ABC}$ 转运蛋白都能利用水解ATP释放的能量直 接转运底物, 其中包括肽、糖、脂、重金属螯合物、多 糖、生物碱、类固醇、无机离子和谷胱苷肽结合物等多 种化合物. 因为 $\mathrm{ABC}$ 转运蛋白在原核和真核生物中与 一些重要的生理过程, 如细菌的耐药性、囊性纤维化和 肿瘤细胞的抗药性等密切相关, 所以引起了人们的广 泛关注 ${ }^{[25,29,40,41]}$. PDR转运蛋白亚家族是 $A B C$ 转运蛋白 家族中的一种新的拓扑构型(NBD-TMD $)_{2}$, 是植物和真 菌中特有的一种转运蛋白 ${ }^{[25]}$.

在植物PDR基因中，有功能报道的基因主要在 第 1 族中, 水生植物浮萍 (Spirodella polyrhiza)的 $S p T U R 2$ 是植物中第一个鉴定的PDR 基因 ${ }^{[42]}, S p T U R 2$ 的表达受 $\mathrm{ABA}$ 以及冷和盐胁迫的诱导, 表明其在非 生物胁迫中的作用 ${ }^{[42,43]}$. 另两个参与非生物胁迫的 是水稻中的OsPDR $9^{[44]}$ 和拟南芥中的AtPDR $12^{[45]}$, 在 根中OsPDR9 的表达受到渗透、盐和缺氧胁迫的强烈 诱导. 其他一些因素, 如重金属 $(\mathrm{Cd}, \mathrm{Zn})$ 和氧化还原 的不平衡同样会增强OsPDR9 的表达. AtPDR12 的表 达受到 $\mathrm{Pb}$ 的强烈诱导, 敲除了 AtPDR12 的植株与野 生型相比, 在含有 $\mathrm{Pb}$ 的营养剂中生长差且体内 $\mathrm{Pb}$ 含 量高. 相反, 过量表达AtPDR12 的植株, 体内 $\mathrm{Pb}$ 含量 低, 表现很强的抗 $\mathrm{Pb}$ 能力. 从烟草细胞系中克隆的 $N P P D R 1$ 基因表现对一种从烟草属植物叶表面发现 的二萜-香紫苏醇的抗性 ${ }^{[46]}$, 诱导 NPPDR1 的表达可 以使细胞获得分泌香紫苏内酯的能力, NPPDR1 可以 在叶表皮毛中表达，其表达可被JA和SA诱导，表明 $N P P D R 1$ 涉及次生代谢物的分泌, 在植物防御中发挥 一定作用 ${ }^{[47]}$. 香紫苏醇还可以诱导 SPTUR2 和 AtPDR12 的表达 ${ }^{[25,48]}$. 现有更多的实验表明 NPPDR1 的这些同源基因可能与生物胁迫和植物防御反应有 关.

$N P P D R 2$ 是在烟草中克隆的, 属于PDR第 4 族, 其转录物存在于根和成熟的花中, 定位于传导组织 中. 在花中, NPPDR2 只在授粉后的花柱中表达. $N p P D R 2$ 不被与生物和非生物胁迫相关的激素诱导 表达, 也不被灰霉病菌 (Botrytis cinerea)诱导表达. 沉默 NpPDR2 的植株未发现任何包括花期的表型变 化, 表明NpPDR2 不是生殖过程必须的 ${ }^{[27]}$. 本研究克 隆的TaPDR1 的表达也不受到JA和SA及非生物胁迫 因子(如热、冷、伤害和 $\mathrm{NaCl}$ )的诱导, 这与 $N P P D R 2$ 的表达模式相同. 但TaPDR1 的表达受到DON和禾谷
镰刀菌的诱导, 表明第 4 族PDR 基因确实参与到生物 胁迫和植物的防御. 香紫苏醇是一种二萜化合物, 被 认为是第 1 族PDR转运蛋白的底物, $\mathrm{DON}$ 是一种倍半 萜化合物, 可能就是第 4 族PDR转运蛋白的底物, 这 对进一步鉴定第 4 族PDR转运蛋白功能有重要的作 用.

PDR5 转运蛋白首先是在酵母 (Saccharomyces cerevisiae)中被发现, 与抗真菌性药物的代谢有关, 是作为排出胞内有毒化合物的洜, 可以将真菌产生 的细胞毒素排出体外, 减少对细胞的毒害, 是一种重 要的抗病蛋白. 酵母 PDR5 基因的突变体对禾谷镰刀 菌产生的细胞毒素DON比野生型更为敏感, 显示了 PDR5 蛋白在抗DON中的作用 ${ }^{[12]}$. 小麦TaPDR1 基因 的表达受到DON和禾谷镰刀菌的诱导, 在抗病品种 中TaPDR1 的表达要比在感病品种中表达早, 并且表 达量高. 由于现在所有报道的PDR转运蛋白均定位在 质膜上, 通过软件预测 $T a P D R 1$ 也定位在质膜上, 所 以推测TAPDR1 可能将真菌毒素DON或DON脱毒产 物从细胞中排出, 减少DON对细胞的毒害, 从而获 得对DON和赤霉病的抗性. 其他的转运蛋白可能负 责向液泡中运输, 因为一些其他的 $\mathrm{ABC}$ 转运蛋白如 TaMDR1 也在本研究所获得的芯片杂交结果中被发 现上调表达.

TaPDR1 除了受到 DON 和禾谷镰刀菌的诱导表 达外, 还受到 $\mathrm{Al}^{3+}$ 和游离 $\mathrm{Ca}^{2+}$ 的诱导表达. 我们推测: ( i ) TaPDR1 没有涉及到铝害, TaPDR1 受到 $\mathrm{Al}^{3+}$ 的诱 导表达是因为铝抑制了钙离子通道, 使细胞内 $\left[\mathrm{Ca}^{2+}\right]$ 下降，诱发了 TaPDR1 表达信号; ( ii ) TaPDR1 既涉及 到 $\mathrm{DON}$ 的抗性, 又对 $\mathrm{Al}$ 害有反应.

动物中发现, DON抑制心肌细胞的钙离子通道 使 $\left[\mathrm{Ca}^{2+}\right]_{\mathrm{i}}$ 下降 ${ }^{[38,39]}$, DON对细胞的最初伤害是抑制了 细胞膜上的钙离子通道. 这对禾谷镰刀菌浸染过程 有重要的生理意义, $\mathrm{Ca}^{2+}$ 是细胞内的第二信使, 广泛 参与了细胞抗病的信号传导. 外源施加JA和 SA都可 以使 $\left[\mathrm{Ca}^{2+}\right]_{\mathrm{i}}$ 增加, 但DON抑制 $\left[\mathrm{Ca}^{2+}\right]_{\mathrm{i}}$ 的增加, 干扰了 抗病信号的传导. 另外, $\mathrm{Ca}^{2+}$ 是细胞膜和细胞壁的重 要组成部分, 钙的亏缺会导致细胞膜和细胞壁的降 解, 这对禾谷镰刀菌菌丝的入侵是很有益的. 小麦对 禾谷镰刀菌的感知和识别在抗感品种中是有明显差 异的, 也同样表现在DON的差异上, $\mathrm{Ca}^{2+}$ 涉及到对 DON抗性的信号传导, 这也可能就是对赤霉病抗性 
的信号传导.

有报道认为, 位于小麦 $3 \mathrm{BS}$ 染色体上的抗赤霉病 和抗DON的主效QTL FHB1 可能就是通过编码或调 控UGTs 基因来实现对DON的抗性 ${ }^{[18]}$, 但迄今为止这
个主效QTL FHB1 还没有被鉴定. 本研究克隆的 $T a P D R 1$ 的表达模式在望水白感病突变体中与在野生 型望水白中明显不同, 显示这个主效QTL FHB1 可能 更像一个调控基因.

\section{参考文献}

1 Windels C E. Economic and social impacts of Fusarium head blight: Changing farms and rural communities in the Northern Great Plains. Phytopathology, 2000, 90: 17-21[DOI]

2 McMullen M P, Jones R, Gallenberg D. Scab of wheat and barley: A re-emerging disease of devastating impact. Plant Dis, 1997, 81: 1340-1348[DOI]

3 Rocha V, Ansari K, Doohan F M. Effects of trichothecene mycotoxins on eukaryotic cells. Food Add Contamants, 2005, 22: 369378[DOI]

4 Boddu J, Cho S, Kruger W M, et al. Transcriptome analysis of the Barley-Fusarium graminearum interaction. Mol Plant Microbe Interact, 2006, 19: 407-417[DOI]

5 Boddu J, Cho S, Muehlbauer G J. Transcriptome analysis of trichothecene-induced gene expression in Barley. Mol Plant Microbe Interact, 2007, 20: 1364-1375[DOI]

6 Mesterhazy A. Types and components of resistance to Fusarium head blight. Plant Breed, 1995, 114: 377-386[DOI]

7 Miller J D, Young J C, Sampson D R. Deoxynivalenol and Fusarium head blight resistance in spring cereals. Phytopathology, 1985, 113: 359-367

8 Miller J D, Arnison P G. Degradation of deoxynivalenol by suspension cultures of the Fusarium head blight resistant wheat cultivar Frontana. Can J Plant Pathol, 1986, 8: 147-150

9 Alexander N J, McCormick S P, Hohn T M. TRI12, a trithothecene efflux pump from Fusarium sporotrichiodes: Gene isolation and expression in yeast. Mol Gen Genet, 1999, 261: 977-984[DOI]

10 Harris L J, Gleddie S C. A modified Rp13 gene from rice confers tolerance of Fusarium graminearum mycotoxin deoxynivalenol to transgenic tobacco. Physiol Mol Plant Pathol, 2001, 58: 173-181[DOI]

11 Lucyshyn D, Busch B L, Abolmaali S, et al. Cloning and characterization of the ribosomal protein L3 (RPL3) gene family from Triticum aestivum. Mol Gen Genet, 2007, 277: 507-517

12 Poppenberger B, Berthiller F, Lucyshyn D, et al. Detoxification of the Fusarium mycotoxin deoxynivalenol by a UDP-glucosyltransferase from Arabidopsis thaliana. J Biol Chem, 2003, 278: 47905-47914[DOI]

13 Rogers B, Decottignies A, Kolaczkowski M, et al. The pleiotropic drug ABC transporters from Saccharomyces cerevisiae. J Mol Biotechnol, 2001, 3: 207-214

14 Somers D J, Fedak G, Savard M. Molecular mapping of novel genes controlling Fusarium head blight resistance and deoxynivalenol accumulation in spring wheat. Genome, 2003, 46: 555-564[DOI]

15 Chen J, Griffey C A, Maroof M A S, et al. Validation of two major quantitative trait loci for Fusarium head blight resistance in Chinese wheat line W14. Plant Breed, 2006, 125: 99-101[DOI]

16 Ma H X, Zhang K M, Gao L, et al. Quantitative trait loci for resistance to Fusarium head blight and deoxynivalenol accumulation in Wangshuibai wheat under field conditions. Plant Pathol, 2006, 55: 739—745[DOI]

17 Jiang G L Dong Y, Shi J R, et al. QTL analysis of resistance to Fusarium head blight in the novel wheat germplasm CJ 9306. II. Resistance to deoxynivalenol accumulation and grain yield los. Theor Appl Genet, 2007, 115: 1043-1052[DOI]

18 Lemmens M, Scholz U, Berthiller F, et al. The ability to detoxify the mycotoxin deoxynivalenol colocalizes with a major quantitative trait locus for Fusarium head blight resistance in wheat. Mol Plant Microbe Interact, 2005, 18: 1318 — 1324[DOI]

19 开增军, 裴自友, 韩航如, 等. 利用 DON test-HPLC 检测小麦镰刀菌毒素 DON 含量的差异. 南京农业大学学报, 2005, 28: 6 $-10$

20 张凯鸣, 马鸿翔, 陆维忠, 等. 小麦赤霉病与 DON 积累的抗性及其相关 SSR 位点差异. 作物学报, 2006, 32: 1788一1795

21 陈怀谷, 蔡志祥, 陈飞, 等. 不同小麦品种抗赤雫病性类型和抗毒素积累能力分析. 植物保护学报, 2007, 34: 32-36

22 Lin F, Kong Z X, Zhu H L, et al. Mapping QTL associated with resistance to Fusarium head blight in the Nanda2419 X Wangshuibai population. I . Type II resistance. Theor Appl Genet, 2004, 109: 1504-1511[DOI]

23 Yu J, Bai G H, Zhou W, et al. Mapping QTLs for different types of resistance to Fusarium head blight in Wangshuibai. Proceedings of the 2005 National Fusarium Head Blight Forum, 2005. 96 
24 Tamura K, Dudley J, Nei M, et al. MEGA4: Molecular evolutionary genetics analysis (MEGA) software version 4.0. Mol Biol Evol, 2007, 24: 1596-1599[DOI]

25 Crouzet J, Trombik O, Fraysse A S, et al. Organization and function of the plant pleiotropic drug resistance ABC transporter family. FEBS Lett, 2006, 580: 1123-1130[DOI]

26 Trombik T, Jasinski M, Crouzet J, et al. Identifi cation of a cluster IV pleiotropic drug resistance transporter gene expressed in the style of Nicotiana plumbaginifolia. Plant Mol Biol, 2008, 66: 165-175[DOI]

27 Kang Z, Buchenauer H. Immunocytochemical localization of Fusarium toxins in infected wheat spikes by Fusarium culmorum. Physiol Mol Plant Pathol, 1999, 55: 275-288[DOI]

28 Krattinger S G, Lagudah E S, Spielmeyer W, et al. A putative ABC transporter confers durable resistance to multiple fungal pathogens in wheat. Sci Exp, 2009, 323: 1360-1363

29 van den Brûle S, Smart C C. The plant PDR family of ABC transporters. Planta, 2002, 216: 95-106[DOI]

30 Sasaki T, Ezaki B, Matsumoto H. A gene encoding multidrug resistance (MDR)-like protein is induced by aluminum and inhibitors of calcium flux in wheat. Plant Cell Physiol, 2002, 43: 177-185[DOI]

31 Kolaczkowski M, van der Rest M, Cybularz-Kolaczkowska A, et al. Anticancer drugs, ionophoric peptides and steroids as substrates of the yeast multidrug transporter Pdr5p. J Biol Chem, 1996, 271: 31543-31548[DOI]

32 Knight H, Trewaras A J, Knight M B. Calcium signaling in Arabidopsis thliana responding to drought and salinity. Plant J, 1997, 12: $1067-1078[\mathrm{DOI}]$

33 Knight H, Trewaras A J, Knight M R. Cold calcium signaling in Arabidopsis involves two cellular pools and a change in calcium signature after acclimation. Plant Cell, 1996, 8: 489-503

34 Knight M R, Anthory KC, Steven M S, et al. Transgenic plant aequorin reports the effects of touch and cold-shock and elicitors on cytoplasmic calcium. Nature, 1991, 352: 524-526[DOI]

35 Gong M, Luit A H, Knight M R, et al. Heal "shock" induced changes in intracellular level in tobacco seedling in reaction to thermotolerance. Plant Physiol, 1998, 116: 429-437[DOI]

36 Kenton P, Mur L A J, Draper J. A requirement for calcium and protein phosphatase in the jasmonate-induced increase in tobacco leaf acid phosphatase specific activity. J Exp Bot, 1999, 50: 1331-1341[DOI]

37 Chinnusamy V, Schumaker K, Zhu J. Molecular genetic perspectives on cross-talk and specificity in abiotic stress signaling in plants. J Exp Bot, 2004, 55: 225-236

38 彭双清, 杨进生. 镰刀菌毒素 DON 对心肌细胞膜电位的影响及硒的保护效应. 中华预防医学杂志, 2003, 37: 423一425

39 彭双清, 杨进生. 镰刀菌毒素脱氧雪腐镰刀菌烯醇对心肌细胞 $\mathrm{Ca}^{2+}$ 通道的阻滞作用. 中国预防医学杂志, 2004, 5: 241一 243

40 Sánchez-Fernández R, Davies T G S, Coleman J O, et al. The Arabidopsis thaliana ABC protein superfamily: A complete inventory . J Biol Chem, 2001, 276: 30231-30244[DOI]

41 Martinoia E, Klein M, Geisler M, et al. Multifunctionality of plant ABC transporters: More than just detoxifiers . Planta, 2002, 214: $345-355[\mathrm{DOI}]$

42 Smart, C C, Fleming A J. Hormonal and environmental regulation of a plant PDR5-like ABC transporter. J Biol Chem, 1996, 271: $19351-19357[\mathrm{DOI}]$

43 van den Brûle S, Muller A, Fleming A J, et al. The ABC transporter SpTUR2 confers resistance to the antifungal diterpene sclareol. Plant J, 2002, 30: 649-662[DOI]

44 Moons A. OsPDR9, which encodes a PDR-type ABC transporter, is induced by heavy metals, hypoxic stress and redox perturbations in rice roots. FEBS Lett, 2003, 553: 370-376[DOI]

45 Lee M, Lee K, Lee J, et al. AtPDR12 contributes to lead resistance in Arabidopsis. Plant Physiol, 2005, 138: 827-836[DOI]

46 Jasinski M, Stukkens Y, Degand H, et al. A plant plasma membrane ATP binding cassette-transporter is involved in antifungal terpenoid secretion. Plant Cell, 2001, 13: 1095-1107

47 Stukkens Y, Bultreys A, Grec S, et al. NpPDR1, a pleiotropic drug resistancetype ATP-binding cassette transporter from Nicotiana plumbaginifolia, plays a major role in plant pathogen defense. Plant Physiol, 2005, 139: 341-352[DOI]

48 Campbell E J, Schenk P M, Kazan K, et al. Pathogen-responsive expression of a putative ATP-Binding Cassette transporter gene conferring resistance to the diterpenoid sclareol is regulated by multiple defense signaling pathways in Arabidopsis. Plant Physiol, 2003, 133: 1272-1284[DOI] 